Mots. Les langages du politique

$94 \mid 2010$

Trente ans d'étude des langages du politique

(1980-2010)

\title{
Le discours politique et son " environnement »
}

Dominique Maingueneau

\section{(2) OpenEdition \\ Journals}

Édition électronique

URL : https://journals.openedition.org/mots/19868

DOI : 10.4000/mots. 19868

ISSN : 1960-6001

Éditeur

ENS Éditions

Édition imprimée

Date de publication : 30 novembre 2010

Pagination : 85-90

ISBN : 978-2-84788-235-3

ISSN : 0243-6450

Référence électronique

Dominique Maingueneau, «Le discours politique et son « environnement » », Mots. Les langages du politique [En ligne], 94 | 2010, mis en ligne le 06 novembre 2012, consulté le 28 avril 2022. URL : http:// journals.openedition.org/mots/19868; DOI : https://doi.org/10.4000/mots.19868 


\section{Mots \\ Les langages du politique}

№ 94 novembre 2010

\section{Trente ans d'étude des langages du politique (1980-2010)}

ouvrage coordonné par Paul BACOT, Marlène Coulomb-GulLY, Jean-Paul Honoré, Christian Le BART, Claire Oger, Christian Plantin

\section{SOMMAIRE}

Paul Bacot, Marlène Coulomb-Gully, Jean-Paul Honoré, Christian Le Bart, Claire Oger, Christian Plantin

Le discours politique n'est pas transparent. Permanence

et transformations d'un objet de recherche

OUTILS ET ENJEUX DU DISCOURS POLITIQUE

Ruth Amossy, Roselyne Koren

Argumentation et discours politique

Christian Plantin

Argumentation-rhétorique. Les eaux mêlées

Caroline Ollivier-Yaniv

Discours politiques, propagande, communication, manipulation 
Marc Bonhomme

La caricature politique

Paul Bacot

Développement et diversification d'une onomastique politique

Ruth Wodak

The Discursive Construction of History. Brief Considerations

Henri Boyer

Les politiques linguistiques

\section{LIEUX DE LA PRODUCTION DU DISCOURS POLITIQUE}

Christian Le Bart

Parler en politique

Dominique Maingueneau

Le discours politique et son «environnement»

Alice Krieg-Planque, Claire Oger

Discours institutionnels. Perspectives pour les sciences

de la communication

Sophie Béroud, Josette Lefèvre

Le corpus syndical. Une expérience au long cours

Corinne Gobin, Jean-Claude Deroubaix

L'analyse du discours des organisations internationales.

Un vaste champ encore peu exploré

François de la Bretèque

Le retour de la parole politique dans le cinéma français

Jean-Claude Soulages

Vie et mort du citoyen cathodique

Jacques Guilhaumou

Les discours de la Révolution française.

Aperçu d'ensemble d'un trajet de recherche (1980-2009) 


\section{DISCIPLINES ET CHAMPS DE RECHERCHE POUR L'ÉTUDE DES LANGAGES DU POLITIQUE}

Philippe Braud

L'apport de la science politique à l'étude des langages du politique

Claire Blandin

L'apport de l'histoire des médias à l'étude des langages du politique

Jean-François Tétu, Bernard Lamizet

Les SIC et les langages du politique

Sylvianne Rémi-Giraud

Sémantique lexicale et langages du politique.

Le paradoxe d'un mariage difficile?

Marlène Coulomb-Gully, Juliette Rennes

Genre, politique et analyse du discours.

Une tradition épistémologique française gender blind

Johannes Angermüller

Analyser le discours politique en Allemagne (1980-2010)

Érik Neveu

L'apport de Pierre Bourdieu à l'analyse du discours.

D'un cadre théorique à des recherches empiriques

Roselyne Ringoot

Questionner le discours avec Michel Foucault.

Actualisations théoriques et actualité éditoriale

\section{ENTRETIEN}

Maurice Tournier

Mots et politique, avant et autour de 1980

La revue Mots. Les langages du politique encourage l'usage des rectifications de l'orthographe proposées par le Conseil supérieur de la langue française et approuvées par l’Académie (Journal officiel, $n^{\circ}$ 100, 6 décembre 1990). 


\section{Le discours politique et son « environnement »}

À la différence de nombreux textes, religieux ou littéraires par exemple, qui sont souvent lus et commentés sans prendre en compte, du moins à un certain niveau, leur contexte sociohistorique, les textes relevant du discours politique ne sont habituellement lus que pour être mis en relation avec un «extérieur» : conjoncture, situation, circonstances... II n'est donc pas surprenant que ces textes aient été le corpus emblématique des débuts de l'analyse du discours en France : procéder à une analyse « interne » de textes que l'on était habitué à ne lire que pour leur « extérieur » constituait une sorte de défi et donnait toute sa force à une entreprise qui visait à lire dans les fonctionnements textuels le «travail» de «l'idéologie». Par la suite, la manière dont on a envisagé les relations entre un «intérieur» et un « extérieur» des textes a changé, en fonction des évolutions de l'analyse du discours.

Dans les débuts de l'analyse du discours française, le seul groupe qui se soit spécialisé de manière durable dans l'étude du discours politique est le laboratoire de lexicométrie de l'ENS de Saint-Cloud, à l'origine de la revue Mots. Les langages du politique ${ }^{1}$. Il a commencé par s'appuyer sur une conception de l'extérieur en termes de visions du monde. Le principe de l'analyse consistait en effet à comparer des corpus qui étaient censés représenter les doctrines politiques d'entités collectives (partis ou groupuscules). Cette démarche s'efforçait donc de maintenir séparés l'«intérieur» et l'«extérieur» du texte : on poussait aussi loin que possible l'analyse immanente de corpus clos pour, dans un second temps, en tirer des conclusions sur les ressemblances/différences entre les groupes qui étaient à sa source.

Ce modèle rassurant a été progressivement mis en cause, de divers points de vue. On évoquera, en premier lieu, les problématiques de l'interdiscours. Désormais l'«extérieur » du texte n'est plus seulement le monde hors du texte, mais aussi des ensembles d'autres textes. Plus précisément, on peut distinguer deux grandes tendances chez ceux qui posent la « primauté de l'interdiscours »:

- l'une, bien illustrée par Michel Pêcheux, qui, en s'appuyant sur la notion

Université Paris-Est Créteil Val de Marne, CEDITEC maingueneau@univ-paris12.fr

1. L'entreprise qui résume le mieux l'ambition du groupe dans les premières années de son existence est celle qui a été menée sur les tracts de Mai 1968. Voir Demonet et al., 1975. 
de «préconstruit », fait du discours l'espace où se nouent l'intradiscours et l'interdiscours ${ }^{2}$ : descriptions définies, nominalisations, relatives... montrent cette présence de l'interdiscours dans l'intradiscours3';

- l'autre qui met en cause la clôture de «formations discursives» référées à tel ou tel locuteur collectif, préférant poser pour unité de base de l'analyse l'interaction entre différentes formations discursives. Cette manière de voir est visible sous des modalités très différentes, chez Jean-Jacques Courtine pour l'étude du discours politique4 et dans mes propres travaux5.

D’autres mises en cause ont été inévitables, sous la pression des courants pragmatiques ou des perspectives qui avaient été ouvertes dès 1969 par L'Archéologie du savoir de Michel Foucault, dont l'influence n'a cessé de croître tout au long des années quatre-vingt. Il est apparu de plus en plus clairement que c'était la distinction même entre un «intérieur» et un «extérieur» du texte politique qui posait problème, que le texte impliquait une activité, le discours, qui était indissociablement linguistique et sociale. On a ainsi vu se multiplier les études sur les genres du discours politique, où « genre » est entendu non pas comme une simple classe où l'on range des textes sur la base de propriétés communes, mais comme des dispositifs de communication à travers lesquels s'articule et se légitime la parole politique en un lieu et à un moment déterminés.

Loin qu'une telle prise en compte de la communication politique interdise l'accès à ce qui serait essentiel, le « contenu » doctrinal des textes, le dispositif d'énonciation apparaît comme la condition, le moteur et aussi l'un des enjeux de l'énonciation. On ne conçoit plus le texte politique comme un simple agencement d' «idées» qui permettrait d' «exprimer» de manière plus ou moins détournée idéologies ou mentalités. Le «contenu» est en réalité traversé par le renvoi à ses conditions d'énonciation. Le contexte n'est pas placé à l'extérieur, dans une série d'enveloppes successives, mais l'énonciation ne fait qu'un avec la gestion même de son contexte. Il n'y a pas, d'un côté, un univers de choses et d'activités muettes, de l'autre, des «représentations » politiques déposées dans des textes.

Une manière de prendre acte de ce déplacement, c'est de raisonner en termes d'institution discursive ${ }^{6}$, en mêlant inextricablement l'institution comme action d'établir, processus de construction légitime, et l'institution au sens usuel d'une organisation de pratiques et d'appareils. Il y a ici enveloppement réciproque : le discours n'advient que s’il se manifeste à travers ces institutions de parole

2. Cette question de l'interdiscours joue un rôle important dans l'ouvrage de Michel Pêcheux, Les Vérités de La Palice (1975), à travers une réflexion sur le préconstruit et les relatives appositives.

3. Sur la problématique de Pêcheux, on se reportera à l'ouvrage de référence édité par Denise Maldidider (1990).

4. Voir à ce propos la réflexion de Jean-Jacques Courtine (1981).

5. Genèses du discours (1984) synthétise les idées défendues dans une thèse de doctorat soutenue à Paris 10 en 1979.

6. Problématique que j'ai développée dans Nouvelles tendances en analyse du discours (1987). 
que sont les genres de discours, pensés à travers les métaphores du rituel, du contrat7, de la mise en scène ${ }^{8}$; de son côté, l'institution politique est sans cesse reconfigurée par les genres de discours qu'elle rend possibles et qui s'autorisent d'elle. Il s'agit donc d'articuler : a) les institutions de divers ordres qui donnent sens à l'énonciation singulière : la structure du champ, le statut de l'acteur politique, les genres de discours... ; b) le mouvement par lequel s'institue le discours, à la fois en instaurant progressivement un certain monde dans son énoncé et en légitimant la scène d'énonciation et le positionnement dans le champ qui rend cette énonciation possible. Si le texte, à travers son énonciation, réfléchit en les légitimant les conditions de sa propre activité énonciative, on comprend quel rôle crucial joue la «scène d'énonciation » que présuppose tout texte; cette scène n'est réductible ni au texte, ni à une situation de communication qu'on pourrait décrire de l'extérieur, comme le ferait un historien ou un sociologue.

Venus d'horizons très différents, en France la théorie des «champs» de Pierre Bourdieu (1976), mais aussi des travaux comme ceux de Michel de Certeau (1974) sur l'historiographie, ont indirectement renforcé l'intérêt pour la dimension «ethnologique» du discours politique, en mettant au centre de la réflexion le fonctionnement des groupes qui le produisent et le gèrent. Le discours politique par nature vise à modifier la société dans son ensemble, mais pour le comprendre, il faut au premier chef le rapporter non à la société considérée dans sa globalité, mais à un secteur limité de cette société, à son «champ », précisément, qui obéit à des règles spécifiques. Un champ est ainsi relativement indépendant des logiques externes, et en particulier des logiques économiques. Les acteurs politiques produisent des textes, mais eux-mêmes et leurs textes sont produits par tout un complexe de pratiques. L'analyste est ainsi amené à faire passer au premier plan les rites des communautés de producteurs de textes politiques, qui sont à la fois la condition et le produit de ces rites 9 .

Le concept de «champ» a permis d'ouvrir des recherches dans deux directions. L'une, qu'on vient d'évoquer, est d'ordre «ethnodiscursif». L'autre permet d'aborder le discours politique en termes de «champ discursif» et de «positionnement », c'est-à-dire comme espace où des producteurs textuels entrent en concurrence pour détenir l'autorité énonciative. Pour clarifier les choses, il nous semble utile de distinguer trois dimensions dans le discours politique.

7. Problématique particulièrement développée par Patrick Charaudeau à travers les concept de «contrat de parole» ou de «contrat de communication » (1983).

8. Erving Goffman (1973) a exploité systématiquement cette métaphore théâtrale, mais sans l'articuler précisément aux genres de discours. Dans mes propres travaux, la scène d'énonciation, à travers la distinction «scène englobante » / «scène générique » / "scénographie », est étroitement liée au concept linguistique de «situation d’énonciation » (Maingueneau, 1998).

9. Ce tournant «ethnodiscursif » était inéluctable, étant donné les présupposés mêmes de l'analyse du discours et la configuration actuelle des sciences humaines et sociales. Il est d'ailleurs encore plus manifeste dans des travaux qui ne s'inscrivent pas dans la tradition d'analyse du discours francophone; en témoigne par exemple le récent livre de Ruth Wodak (2009). 
Certes, il se présente comme un champ, un lieu de confrontation entre des positionnements politiques qui investissent, selon leurs modes propres, des genres de discours. Mais c'est aussi :

- un réseau d'appareils où des individus peuvent se constituer en acteurs légitimes, où sont stabilisés et garantis les contrats des genres de discours considérés comme politiques, où interviennent des médiateurs (journalistes...), des interprètes et des évaluateurs autorisés (commentateurs, enseignants...), des canons (stabilisés dans des manuels, des formations professionnelles...);

- une archive où se mêlent textes et légendes : la Commune, l'Appel du 18 juin, Mai 1968... L'activité politique est plongée dans cette mémoire qui, en retour, est elle-même prise dans les conflits du champ, qui ne cessent de la retravailler.

Un autre type d' «environnement» des textes politiques, étroitement lié à la problématique des genres, c'est tout ce qui concerne les supports matériels du discours, leur dimension «médiologique», pour reprendre le terme de Régis Debray (1991). L'analyse du discours politique est apparue dans les années soixante, dans une société qui était encore dominée par l'écrit et dans une conjoncture où une part essentielle de l'activité politique consistait à lire de grands auteurs, avec tous les conflits d'interprétation que cela impliquait. N'oublions pas que l'œuvre majeure d'Althusser s'appelait Lire le Capital ${ }^{10}$. Le renforcement de l'emprise de la télévision, puis le développement fulgurant d'Internet ont considérablement modifié les conditions de l'énonciation politique et les relations entre le discours politique et les citoyens. Cette transformation a eu un double effet : d'une part, il s'est produit un brouillage de la frontière entre les études sur les médias et les études sur le discours politique (le fameux duel qui clôt la campagne présidentielle est à la fois un genre politique et un genre télévisuel); d'autre part, on a modifié le regard porté sur l'exercice antérieur de la parole politique, en particulier en ce qui concerne l'oralité rhétorique, l'importance de la radio, de la presse industrielle..., réalités aussi massives que discrètes qui ont informé en profondeur le dicible politique.

Pour terminer, j’aimerais évoquer une problématique plus personnelle. En général, quand on travaille sur le discours politique, on le considère en luimême, ou alors dans ses relations avec tel ou tel de ses voisins (politique/ religion, politique/médias, politique/science...). Pourtant, ce discours ne prend sens qu'inscrit dans l'ensemble des discours d'une société. L'une des manières de gérer cette difficulté est de situer le discours politique par rapport aux « discours constituants $»^{11}$. Le discours politique n'est pas un discours constituant, comme peuvent l'être le discours philosophique, le discours reli-

10. En fait, c'est un ouvrage collectif de Louis Althusser, Étienne Balibar, Roger Establet, Pierre Macherey et Jacques Rancière (1965).

11. Voir Maingueneau et Cossutta (1995), Maingueneau (1999). 
gieux ou le discours scientifique, qui se fondent de manière réflexive, mais il se situe à la jointure entre ces discours constituants et les multiples niveaux de la doxa, de l'opinion commune. Les positionnements politiques sont ainsi obligés de s'appuyer dans des proportions variables sur les discours constituants, en faisant prédominer tel ou tel : le discours religieux pour les théocraties, le discours scientifique pour les technocrates, la philosophie des Lumières pour la République française, etc. Mais la mise à mort du Christ ou de Socrate est là pour nous rappeler que si le politique doit s'appuyer sur des discours constituants, il les domine aussi, dès lors qu'il dispose du pouvoir.

Quand on cherche à comprendre comment l'analyse du discours politique a géré la relation du politique à son "environnement», on voit à quel point les choses se sont complexifiées depuis la fin des années soixante. Pour autant, on ne disposera pas ces divers apports théoriques et méthodologiques sur une ligne de «progrès », en disqualifiant à chaque fois l'étape précédente. En fait, il vaut mieux considérer qu'on a aujourd'hui une vision plus réaliste de la complexité et de l'hétérogénéité de ce type de discours et des multiples approches qu'il rend possibles. On ne considérera pas, par exemple, qu'une approche lexicométrique doit s'effacer devant une approche en termes de champ discursif ou de genre de discours. Ou encore qu'une étude des textes de doctrine est obsolète, l'essentiel étant les modes de mise en scène de la parole politique. Dans la mesure où la manière dont on conçoit le discours politique a changé, les diverses approches ne peuvent plus avoir la même signification. Chacune est condamnée à prendre la mesure de ses limites autant que de ses pouvoirs.

\section{Références}

Althusser L., Balibar É., Establet R., Macherey P., Rancière J., 1965, Lire le Capital, Paris, Maspero.

BouRdieu P., 1976, "Le champ scientifique», Actes de la recherche en sciences sociales, n²-3, p. 88-104.

Certeau M. (de), 1975, L'écriture de l'histoire, Paris, Gallimard.

Charaudeau P., 1983, Langage et discours. Éléments de sémiolinguistique, Paris, Hachette, 1983.

COURTINE J.-J., 1981, «Quelques problèmes théoriques et méthodologiques en analyse du discours. À propos du discours communiste adressé aux chrétiens », Langages, n०62, p. 9-127.

DeBray R., 1991, Cours de médiologie générale, Paris, Gallimard.

Demonet M., Gouaze J., Lafon P., Moulllaud M., Tournier M., 1975, Des tracts en mai 1968, Paris, Armand Colin (réédition 1978, Paris, Champ Libre).

Goffman E., 1973, La mise en scène de la vie quotidienne, I, La présentation de soi, Paris, Minuit. 
Maingueneau D., 1984, Genèses du discours, Liège, Mardaga.

- 1987, Nouvelles tendances en analyse du discours, Paris, Hachette.

- 1998, Analyser les textes de communication, Paris, Dunod.

- 1999, "Analysing self-constituting discourses», Discourse studies, vol.l, n² 2 , p. 175-199.

Maingueneau D., Cossutta F., 1995, "L'analyse des discours constituants», Langages, $\mathrm{n}^{0} 117, \mathrm{p} .112-125$.

Maldidier D. éd., 1990, L'inquiétude du discours. Textes de Michel Pêcheux, Paris, Éditions des Cendres.

PÊCheux M., 1975, Les Vérités de La Palice, Paris, Maspéro.

WodAK R., 2009, The Discourse of Politics in Action. Politics as Usual, New York, Palgrave Macmillan. 